Guerra Química: Perspectivas no Estudo de Reativadores da Enzima Acetilcolinesterase Inibida por Organofosforados

\author{
Giacoppo, J. O. S.; Lima, W. E. A.; Kamil, K.; França, T. C. C.; da Cunha, E. \\ F. F.; Ramalho, T. C.*
}

Rev. Virtual Quim., 2014, 6 (3), 653-670. Data de publicação na Web: 13 de abril de 2014

http://www.uff.br/rvq

\title{
Chemical Warfare: Perspectives on Reactivating the Enzyme Acetylcholinesterase Inhibited by Organophosphates
}

\begin{abstract}
Recent attacks on Syria using the nerve agent sarin warned once again the world and the scientists on the use of chemical weapons. It is known that nerve agents are potent inhibitors of acetylcholinesterase (AChE), the enzyme responsible for the hydrolysis of the neurotransmitter acetylcholine and, thus, transmitting of nerve impulses. The process of AChE inhibition by organophosphate (OP) can be reversed by a nucleophile, responsible for dephosphorylation of the residue Ser203 in the active site of the enzyme. In this sense, oximes exhibit this characteristic and are able to remove the neurotoxic and reactivate the enzyme. Here, we review experimental and theoretical results involving docking and quantum mechanical-molecular mechanics hybrid methods (QM/MM), using Molegro ${ }^{\circledR}$ and Spartan $^{\circledR}$ softwares to analyze the interaction of different OPs and oximes with AChE and to evaluate kinetic constants of reactivation.
\end{abstract}

Keywords: Chemical warfare; organophosphates; oximes; docking; QM/MM.

\section{Resumo}

Os ataques recentes na Síria utilizando o agente neurotóxico sarin alertaram mais uma vez a opinião pública mundial e a comunidade científica quanto à utilização de armas químicas. Sabe-se que agentes dos nervos são potentes inibidores da acetilcolinesterase (AChE), enzima responsável pela hidrólise do neurotransmissor acetilcolina e, consequentemente, pela transmissão de impulsos nervosos. A inibição da AChE por um organofosforado (OP) pode ser revertida por um nucleófilo, que desfosforila a Ser203 no sítio ativo da enzima. Nesse sentido, as oximas têm essa característica, pois são capazes de remover o neurotóxico e reativar a enzima. Neste trabalho, revisamos alguns resultados experimentais e teóricos que envolvem estudos de ancoramento molecular e métodos híbrídos quanto-mecânicos/mecânica molecular (QM/MM) usando os softwares Molegro ${ }^{\circledast}$ e Spartan ${ }^{\circledast}$ para analisar as interações de diferentes oximas e OPs com a AChE e avaliar as constantes cinéticas de reativação.

Palavras-chave: Guerra química; organofosforados; oximas; ancoramento; QM/MM.

* Universidade Federal de Lavras, Laboratório de Química Computacional, Departamento de Química, CEP 37200-000, Lavras-MG, Brasil.

Mteo@dqi.ufla.br

DOI: $\underline{10.5935 / 1984-6835.20140041}$

Rev. Virtual Quim. |Vol 6| | No. 3| |653-670| 


\section{Guerra Química: Perspectivas no Estudo de Reativadores da Enzima Acetilcolinesterase Inibida por Organofosforados}

Juliana de O. S. Giacoppo, ${ }^{a}$ Willian E. A. de Lima, ${ }^{a}$ Kamil Kuca, ${ }^{b, c}$ Elaine F. F. Cunha, ${ }^{a}$ Tanos C. C. França, ${ }^{d}$ Teodorico de C. Ramalho ${ }^{a, *}$

a Universidade Federal de Lavras, Departamento de Química, CP 3037, CEP 37200-000, Lavras, MG, Brasil.

${ }^{\mathrm{b}}$ University of Defense, Center of Advanced Studies, Faculty of Military Health Sciences UO, Czech Republic.

${ }^{\mathrm{c}}$ University Hospital Hradec Kralove, Biomedical Research Center, Hradec Kralove, Czech Republic.

dInstituto Militar de Engenharia, Laboratório de Modelagem Molecular Aplicada à Defesa Química e Biológica (LMDQB), CEP 22290-270, Rio de Janeiro-RJ, Brasil.

\section{* teo@dqi.ufla.br}

\section{Introdução}

1.1. Guerra Química

1.2. Os neurotóxicos

\section{Procedimento Experimental}

2.1. Estudos por ancoramento molecular

2.2. Os métodos híbridos-QM/MM

\section{Resultados e Discussão}

3.1. Estudo das interações entre a oxima $\mathrm{BI}-6$ e a $\mathrm{AChE}$ inibida por diferentes OPs

3.2. Estudos das interações entre diferentes oximas e a AChE inibida por ciclosarin

\section{Considerações finais}

\section{Introdução}

\subsection{Guerra química}

Armas de destruição em massa são aquelas que, quando utilizadas, têm a capacidade de provocar um grande número de mortes em uma única ação. ${ }^{1}$ Recebem essa designação as armas nucleares, químicas e biológicas, as quais representam uma ameaça quase inimaginável para a sociedade. $^{2}$

Os agentes de guerra química e biológica constituem uma classe de armas não 
convencionais de baixo custo, mas de difícil detecção e controle., ${ }^{3,4}$ Não há grandes obstáculos técnicos na produção desses agentes, como acontece no caso das armas nucleares. A cultura de agentes biológicos em laboratório, por exemplo, é feita com técnicas básicas de microbiologia, utilizando materiais de amplo emprego e fácil aquisição.

Amostras desses micro-organismos são rotineiramente comercializadas para fins de pesquisa de vacinas e medicamentos, podendo também, em certos casos, serem obtidas diretamente de animais infectados na natureza. ${ }^{6}$ Agentes químicos, por sua vez, podem ser produzidos em grande escala em praticamente qualquer instalação industrial.

A expressão "guerra química" foi utilizada pela primeira vez em 1917 em função do uso de agentes químicos em larga escala nas trincheiras da primeira guerra mundial. ${ }^{7,8}$ De acordo com a Convenção para Prevenção de Armas Químicas (CPAQ), ${ }^{9}$ agentes químicos de guerra são definidos como substâncias químicas, quer gasosas, líquidas ou sólidas, que podem ser utilizadas devido aos seus efeitos tóxicos diretos sobre seres humanos, animais ou plantas. ${ }^{10} \mathrm{Em}$ um contexto militar, pode-se mencionar dois objetivos básicos para o uso de armas químicas: provocar baixas (mortos e feridos) e diminuir o desempenho operacional das tropas inimigas pela necessidade do uso prolongado de equipamentos de proteção. ${ }^{10}$

Devido à facilidade de manufatura e ao baixo custo, as armas químicas passaram do interesse estritamente militar para 0 de organizações terroristas. Isso faz com que a defesa contra a guerra química deixe de ser um objeto de preocupação essencialmente militar e torne-se uma preocupação também do meio civil. ${ }^{9,11}$

\subsection{Os neurotóxicos}

Os organofosforados (OP) são compostos químicos com bastante importância militar. Eles são inibidores da acetilcolinesterase (AChE) extremamente tóxicos. ${ }^{14}$ Estes compostos inibem a atividade da AChE nas sinapses ligando um grupo fosforila a seu sítio ativo, resultando no acúmulo do neurotransmissor acetilcolina (ACh) e ativação descontrolada das sinapses colinérgicas. ${ }^{15}$

Os agentes de nervos (ou neurotóxicos) são o grupo mais letal dentre os OPs. Tabun (etil $N, N$-dimetilfosforamidocianidato), sarin [2-(fluoro-metil-fosforil)oxipropano], soman [2-(fluoro-metil-fosforil)oxi-3,3-dimetil-

butano], ciclosarin [(Fluoro-metilfosforil)oxiciclohexano] e VX $\{S$-[2(diisopropilamino) etil]-O-etil metilfosfonotioato\} (Figura 1) são, provavelmente, os membros mais conhecidos dessa família. ${ }^{16-20}$ Esses compostos têm como alvo principal a AChE. ${ }^{17,21}$

Recentemente, um ataque na Síria (agosto de 2013) com o neurotóxico sarin alertou mais uma vez a opinião pública mundial e a comunidade científica para a utilização de armas químicas. Estima-se que foram mortas mais de 1.400 pessoas, dentre elas 426 crianças. Segundo os médicos sírios o que contribuiu para alta mortalidade foram as quantidades limitadas de antídotos \{pralidoxima [(2-hidroxiimino)metil]-1metilpiridin-1-ium] e diazepam [7-cloro-1,3dihidro-1-metil-5-fenil-1,4-benzodiazepin$2(2 \mathrm{H})$-ona)]\} disponíveis para o tratamento. ${ }^{16}$

Em geral, como se pode ver na Figura 1, os neurotóxicos têm um átomo de fósforo quiral ligado a um grupo de saída $X$ (como o flúor ou o cianeto, por exemplo), a um grupo R1 (alquila ou dialquilamino) e um grupo $R$ [alquila, cicloalquila, $\mathrm{H}$ (somente na série $\mathrm{V}$ ), $\left(\mathrm{CH}_{2}\right)_{n} \mathrm{~N}^{+} \mathrm{R}_{3}$ (quando $\mathrm{X}=\mathrm{F}$ )], em ligação dupla com um grupo $Y$ (oxigênio ou, mais raramente, enxofre). 


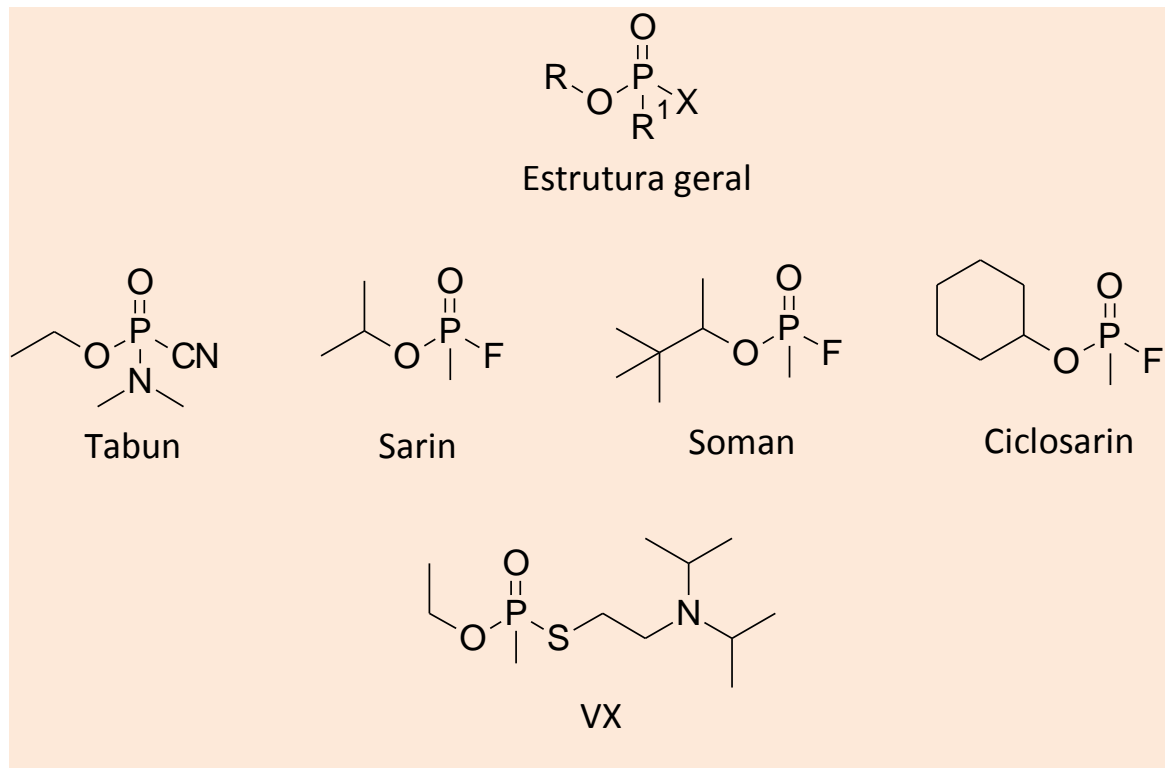

Figura 1. Estruturas de alguns neurotóxicos

Os OPs reagem diretamente com a hidroxila da serina da tríade catalítica do sítio ativo da AChE e formam um complexo relativamente estável (OP-AChE), levando a efeitos tóxicos fatais. ${ }^{22}$ Esses compostos provocam o colapso do sistema nervoso central. Os sintomas são angústia, perda da coordenação e convulsões que levam à morte. $^{23}$

Atualmente, o tratamento padrão contra o envenenamento por OP inclui a utilização de um anticolinérgico, normalmente atropina intravenosa, para diminuir os efeitos da acumulação da ACh, combinada com um depressor do sistema nervoso central, como - diazepam, para reduzir convulsões e espasmos, e uma oxima catiônica para reativar a AChE. Esses três componentes são combinados em seringas autoinjetoras que podem ser levadas para o campo de batalha. ${ }^{5,21,24}$

A AChE ou acetilcolina acetil-hidrolase, é uma enzima regulatória responsável pela transmissão dos impulsos nervosos nas sinapses por meio da hidrólise da ACh, uma função chave na regulação da transmissão dos impulsos nervosos que, se inibida, geralmente, leva rapidamente à morte..$^{25,14}$

Essa enzima está presente nos sistemas nervosos central e periférico, sobre o receptor de $\mathrm{ACh}$ e na transmissão de impulsos nervosos, por meio das sinapses neuromusculares. É uma das enzimas mais eficientes, sendo capaz de hidrolisar rapidamente a ACh nas junções neuromusculares e nas sinapses colinérgicas no cérebro. ${ }^{26,27}$

Cada monômero da AChE contém um centro catalítico composto por dois compartimentos: o subsítio catalítico, a tríade catalítica e o subsítio aniônico, que acomoda o nitrogênio com carga positiva da molécula de $\mathrm{ACh} .{ }^{14}$ Nessas regiões há quatro domínios, sendo que no primeiro encontramse os resíduos de serina e histidina da tríade catalítica (Ser203, Glu334 e His447 na enzima humana), que é encontrada no fundo da "garganta" do sítio ativo. ${ }^{26,28}$ O segundo é o próprio subsítio aniônico, localizado a uma distância igual ou superior a 4,7 $\AA$ da hidroxila da Ser203, no qual o grupo amônio da ACh interage, eletrostaticamente, com o Glu334. ${ }^{28,29}$ O papel do subsítio aniônico é orientar a parte carregada do substrato que entra no sítio ativo. ${ }^{14} \mathrm{O}$ terceiro domínio é constituído por uma região hidrofóbica importante para a ligação com substratos cíclicos. ${ }^{28,29}$ Existe, ainda, um quarto domínio na $\mathrm{AChE}$, no qual interagem ligantes 
catiônicos e alguns ligantes neutros. Esse domínio se localiza a mais de $20 \AA$ do sítio ativo e, por isso, é denominado sítio aniônico periférico, consistindo (na enzima humana) dos resíduos Asp74 e Trp286 como um núcleo comum.

É importante ressaltar que a complexação de ligantes com o sítio periférico provoca frequentemente alterações na conformação do sítio ativo. As interações dos ligantes com esses resíduos podem ser a chave para a modulação alostérica da atividade catalítica da AChE. ${ }^{14,28,30}$

Devido à função chave da $\mathrm{AChE}$, ela se tornou um dos alvos moleculares mais vulneráveis a agentes neurotóxicos, pesticidas e venenos de cobras, e, também, a fármacos voltados para o tratamento de doenças como o mal de Parkinson e a doença de Alzheimer. ${ }^{28,29}$

Alguns OP formam conjugados de fósforo ligados por covalência ao resíduo Ser203 que interferem no mecanismo catalítico da AChE. Esses compostos impedem a hidrólise da ACh, obstruindo a transmissão colinérgica. ${ }^{26,28,29}$ Após a inibição da AChE por um OP (Figura 2), podem acontecer dois processos: o envelhecimento ou a reativação espontânea da enzima, processo que pode ser acelerado em várias ordens de grandeza pela adição de um nucleófilo forte como uma oxima. ${ }^{31}$ No entanto, sem a presença de oximas, a reação de reativação para a maior parte dos complexos AChE-OP ocorre a uma taxa insignificante, o que torna a reação de envelhecimento majoritária. ${ }^{26} \mathrm{O}$ processo de envelhecimento ocorre por uma reação de eliminação ou pela desalquilação espontânea do fósforo conjugado na $\mathrm{AChE}$, com quebra da ligação oxigênio-alquila, resultando em um complexo aniônico bastante estável, resistente à reação de reativação por oximas conhecidas atualmente e fazendo com que a enzima seja inibida irreversivelmente. ${ }^{26,31}$

A reativação da $A C h E$ se baseia na desfosforilação da Ser203. Esse é o principal mecanismo de ação das oximas. Se a oxima possui nitrogênio ligado ao anel aromático ocorre, durante o processo de reativação, a atração da região com carga positiva da oxima pelo sítio aniônico da $\mathrm{AChE}$, permitindo, assim, que a parte ativa da oxima se posicione sobre o sítio fosforilado da AChE e promova o ataque nucleofílico, como pode ser visto na Figura $2 .^{22,32}$

É importante destacar que a eficiência dessa reação de reativação depende de fatores como as estruturas químicas do OP e do reativador (oxima). Os complexos formados entre a AChE e o neurotóxico tabun, por exemplo, exibem alta resistência à reativação, enquanto os complexos formados com os neurotóxicos $\mathrm{VX}$ e sarin podem ser reativados por uma série de diferentes substâncias. ${ }^{20}$

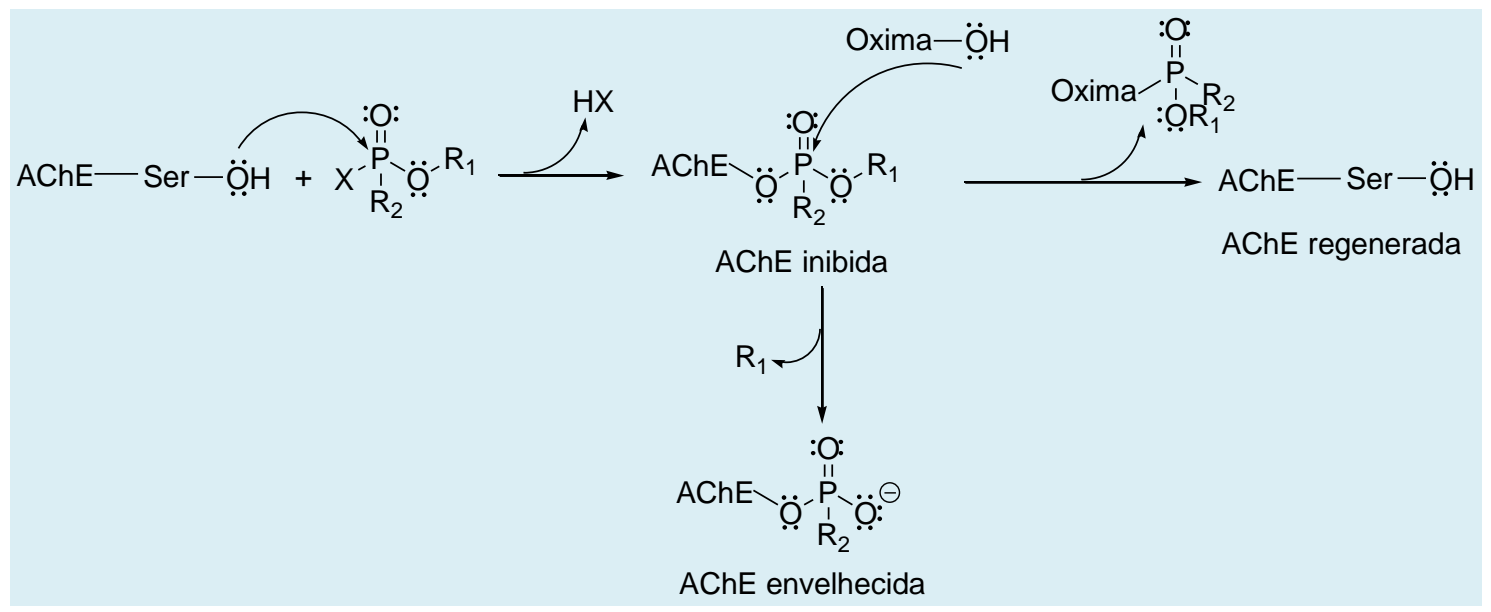

Figura 2. Processo de inibição e reativação da $A C h E . R_{1}=$ grupo alquila, $O$-alquila ou amida; $R_{2}$ = grupo O-alquila ou amida; e $\mathrm{X}=$ grupo de saída 
No século passado, várias oximas foram utilizadas como antídotos contra agentes neurotóxicos. Porém, infelizmente, ainda não se encontrou uma oxima universal que seja eficiente com qualquer neurotóxico. ${ }^{35,36}$ Isto confirma a importância da busca por novas oximas mais eficazes contra esses agentes.

Neste trabalho são revisados alguns resultados obtidos em nosso grupo através de estudos experimentais e teóricos (ancoramento molecular e métodos híbrídos quanto-mecânicos/mecânica molecular (QM/MM) em duas linhas de pesquisa: Uma visa a reativação, pela oxima BI-6 (1-2hidroxiiminometilpiridinium-4-

carbamoilpiridinium-2-buteno), da AChE inibida por diferentes $\mathrm{OPs}^{37}$ e a outra trata da reativação por diferentes oximas da AChE inibida por ciclosarin. ${ }^{23}$

\section{Procedimento Experimental}

\subsection{Estudos por ancoramento molecular}

A simulação computacional por ancoramento molecular é uma das mais importantes técnicas de investigação das interações moleculares entre uma proteína e um ligante, nos casos em que a estrutura 3D da proteína já foi elucidada ${ }^{38}$, seja por cristalografia, por RMN ou por modelagem comparativa (homologia). ${ }^{6,39,40}$

Os valores da função de desempenho do ancoramento $E_{\text {score }}$ são definidos pelas Equações 1, 2 e 3.

$$
\begin{gathered}
E_{\text {score }}=E_{\text {inter }}+E_{\text {intra }} \\
E_{\text {inter }}=\sum_{i \in \text { ligante }} \sum_{j \in \text { proteina }}\left[E_{P L P}\left(r_{i j}\right)+332.0 \frac{q_{i} q_{j}}{4 r_{i j}^{2}}\right]
\end{gathered}
$$

$E_{\text {inter }}$ corresponde à energia de interação ligante-proteína e o termo $E_{P L P}$ representa a energia potencial do inibidor, que usa dois conjuntos diferentes de parâmetros: um para a aproximação do termo estérico (van der Waals) entre átomos e outro potencial para a ligação hidrogênio. $O$ segundo termo descreve as interações eletrostáticas entre átomos carregados. É um potencial de Coulomb com uma constante dielétrica dependente da distância $(D(r)=4 r)$. O valor numérico de 332,0 fixa as unidades de energia eletrostática em $\mathrm{kcal}^{\mathrm{mol}^{-1}}{ }^{41,42,43} \mathrm{e}$ $E_{\text {intra }}$ é a energia interna do ligante (Equação $3)$.

$$
\text { Eintra }=\sum_{\text {ieligante }} \sum_{j \in \text { ligante }} E_{P L P}\left(r_{i j}\right)+\sum_{\text {ligaçöesflexiveis }} A\left[1-\cos \left(m . \theta-\theta_{0}\right)\right]+E_{\text {penalizada }} \quad \text { Eq. } 3
$$

As duas primeiras somas contidas na equação 3 estão relacionadas a todos os pares de átomos do ligante, excluindo-se os pares de átomos conectados por duas ligações. $O$ segundo termo refere-se à energia de torção, em que $\theta$ é o ângulo de torção da ligação. $O$ último termo, $E_{\text {penalizada }}$ (chamado de termo de correção), atribui uma penalidade de 1.000 se a distância entre dois átomos pesados (mais de duas ligações distantes) for menor do que 2,0 $\AA$, punindo conformações inexistentes do ligante. Em resumo, essas funções são utilizadas para 
ancorar automaticamente uma molécula flexível em uma molécula molde (proteína). ${ }^{41}$

É importante ter em mente que as moléculas, geralmente desenhadas de forma tridimensional não estão, necessariamente, na conformação mais estável. Durante a geração de uma determinada estrutura ocorrem distorções na molécula com formação desfavorável de comprimentos e ângulos de ligações e ângulos diédricos. Átomos não-ligados também interagem em uma mesma região do espaço e provocam repulsão estérica e eletrostática. Para corrigir estas distorções as moléculas são otimizadas pelo processo de minimização de energia, a partir de dois modelos matemáticos (i) mecânica molecular ou (ii) mecânica quântica. Interações não previsíveis, relacionadas à sobreposição de orbital molecular, distribuição de densidade eletrônica, ou interferências estéricas podem ser solucionadas pelos métodos computacionais. A minimização de energia e a análise conformacional são usadas interativamente, para otimizar a geometria de uma molécula. ${ }^{20}$ Entretanto para entender o mecanismo de interação de um fármaco é essencial saber 0 posicionamento tridimensional para a sua interação molecular com a proteína alvo. A predição da geometria e da energia de ligação é de grande interesse para o planejamento de novos medicamentos. Neste sentido, a técnica de ancoramento molecular pode ser extremamente útil. De fato, essa técnica encontra uma média de estruturas estáveis do ligante na proteína e calcula essa estabilidade relativa. Para encontrar a estrutura de menor energia, sem qualquer suposição prévia, é necessário analisar todos os modos de interação, considerando a flexibilidade conformacional do ligante a ser introduzido no sítio ativo da proteína. Como esses dois problemas estão interligados, eles podem ser resolvidos ao mesmo tempo. Contudo, o número de combinações envolvidas é muito grande. ${ }^{44}$ Apesar da grande importância e das inúmeras aplicações, a técnica de ancoramento molecular é limitada se o fenômeno envolver quebra e formação de ligações químicas. Neste caso, aplica-se técnicas híbridas de $\mathrm{QM} / \mathrm{MM}$.

\subsubsection{Estudo das interações entre a oxima} BI-6 e a AChE inibida por diferentes OPS

Os estudos in vitro da $\mathrm{BI}-6$ envolveram uma coleção de procedimentos experimentais padronizados. ${ }^{45}$ Cérebros de ratos foram utilizados como fonte de AChE. Eles foram obtidos de ratos anestesiados (a narcose não influencia a atividade da $\mathrm{AChE}^{16}$ ) após decapitação e homogeneização em água. A eficiência da reativação foi testada com homogenato de cérebro de rato $(p / v)$ que foi incubado com o agente neurotóxico apropriado por 30 minutos para atingir $96 \%$ de inibição da AChE. Após a Bl-6 foi adicionada por 10 minutos. As medidas foram feitas a 25 oC, pH 8 e a concentrações de entre $10-3$ e 10-5 M. As atividades da AChE foram medidas por um método potenciostático utilizando um titulador RTS 822 (Radiometer, Denmark). Os dados sobre a taxa inicial (i.e parte inicial da curva) da reação da enzima com o substrato (iodeto de acetilcolina 0,02 M) tornaram possível o cálculo do percentual de ativação a partir da equação 2 .

$$
\% \text { reativação }=100-100(a o-a r) /(a o-a i) \quad \text { Eq. } 4
$$

onde $a_{0}$ é a atividade da enzima intacta, $a_{i}$ é a atividade da enzima inibida pelo OP e $a_{r}$ é a atividade da enzima inibida pelo OP após incubação com BI-6.

As coordenadas cristalográficas da AChE de Mus Musculus (MmAChE) fosforilada por sarin e complexada com a oxima HI-6 (4carbamoil-1-[(\{2-[(E)-

(hidroxiimino)metil]piridinium-1-

il\}metoxi)metil]piridinium dicloreto) foram 
retiradas do Protein Data Bank (código PDB: 2WHP). ${ }^{46}$ e a estrutura da HI-6 em seu interior foi modificada para a oxima BI-6 utilizando o software Spartan $08^{\circledR},{ }^{\circledR 7}$ a fim de se obter a estrutura 3D da AChE complexada com BI-6 necessária para os estudos deste trabalho.

As estruturas tridimensionais dos OPs sarin, ciclosarin, soman e VX (Figura 1) foram construídas com base na estrutura do sarin presente no cristal, também usando o programa Spartan08 ${ }^{\circledR} .^{47}$ Subsequentemente, as otimizações de geometria e os cálculos de distribuição de carga atômica parciais foram realizados com o mesmo programa, utilizando o método de orbital molecular semi-empírico AM1.

Primeiramente foi realizado o estudo de ancoramento molecular dos OPs no sítio ativo da MmAChE a fim de se obter as conformações mais estáveis destes compostos. Observe-se que, para isso, o sarin foi selecionado como ligante ativo. Após a análise dos resultados, foram selecionadas as melhores poses. Para a realização dos cálculos de ancoramento e re-docking, foi utilizado o programa Molegro ${ }^{\circledR}$ Virtual Docker 2006 (MVD). ${ }^{41}$ A partir das simulações de docking realizadas entre cada composto estudado e a enzima, foram selecionadas as poses com as menores energias obtidas.

Os cálculos foram realizados de acordo com as instruções do programa. Os sítios de ligação foram restringidos dentro de uma esfera com raio de $7 \AA$, centralizada na estrutura da oxima $\mathrm{BI}-6$ e incluindo todos os resíduos do sítio ativo. Devido à natureza estocástica do algoritmo de busca do ancoramento ligante-proteína, cerca de 15 soluções de ancoramento foram retidas para cada ligante. As poses com melhor sobreposição em relação à HI-6 presente no cristal foram escolhidas para a análise realizada neste trabalho.

2.1.2. Estudos das interações entre diferentes oximas e a AChE inibida por ciclosarin

Os dados in vitro de constante de ligação oxima/enzima inibida $\left(K_{R}\right)$ e constante de velocidade do processo de reativação $\left(k_{R}\right)$ para as oximas estudadas neste trabalho (Figura 3) relativos a $\mathrm{MmAChE}$ inibida por ciclosarin foram reportados por Kassa et al. (2007). ${ }^{48}$

As coordenadas cristalográficas da MmAChE fosforilada por sarin e complexada com a oxima HI-6 (PDB código: 2WHP) foram retiradas do Protein Data Bank $(P D B)^{46}$ e tiveram suas moléculas de água cristalográficas removidas com o software SPDBViewer. ${ }^{49}$ A estrutura do sarin dentro da MmAChE foi completada pela introdução do grupo $-\mathrm{CH}_{2} \mathrm{CH}_{2} \mathrm{CH}_{2}-$ usando $\mathrm{O}$ software Spartan08 ${ }^{{ }{ }^{47}}$, afim de se obter a estrutura 3D da MmAChE fosforilada por ciclosarin necessária para a realização do trabalho. As estruturas 3D de cada oxima apresentada na Figura 3 foram construídas com base na estrutura da oxima HI-6 em sua conformação bioativa presente no cristal.

As oximas foram ancoradas no sítio ativo da MmAChE usando o $\mathrm{MVD}^{\circledR}{ }^{41}$ de acordo com as instruções do programa. Os sítios de ligação foram restringidos dentro de uma esfera com raio entre 9 e $15 \AA$, de acordo com o tamanho do ligante, centralizada na estrutura da HI-6 e incluindo todos os resíduos do sítio ativo. Cerca de 30 soluções de ancoramento foram obtidas para cada ligante. As poses com melhor sobreposição em relação à HI-6 do cristal foram escolhidas para a análise realizada neste trabalho. 


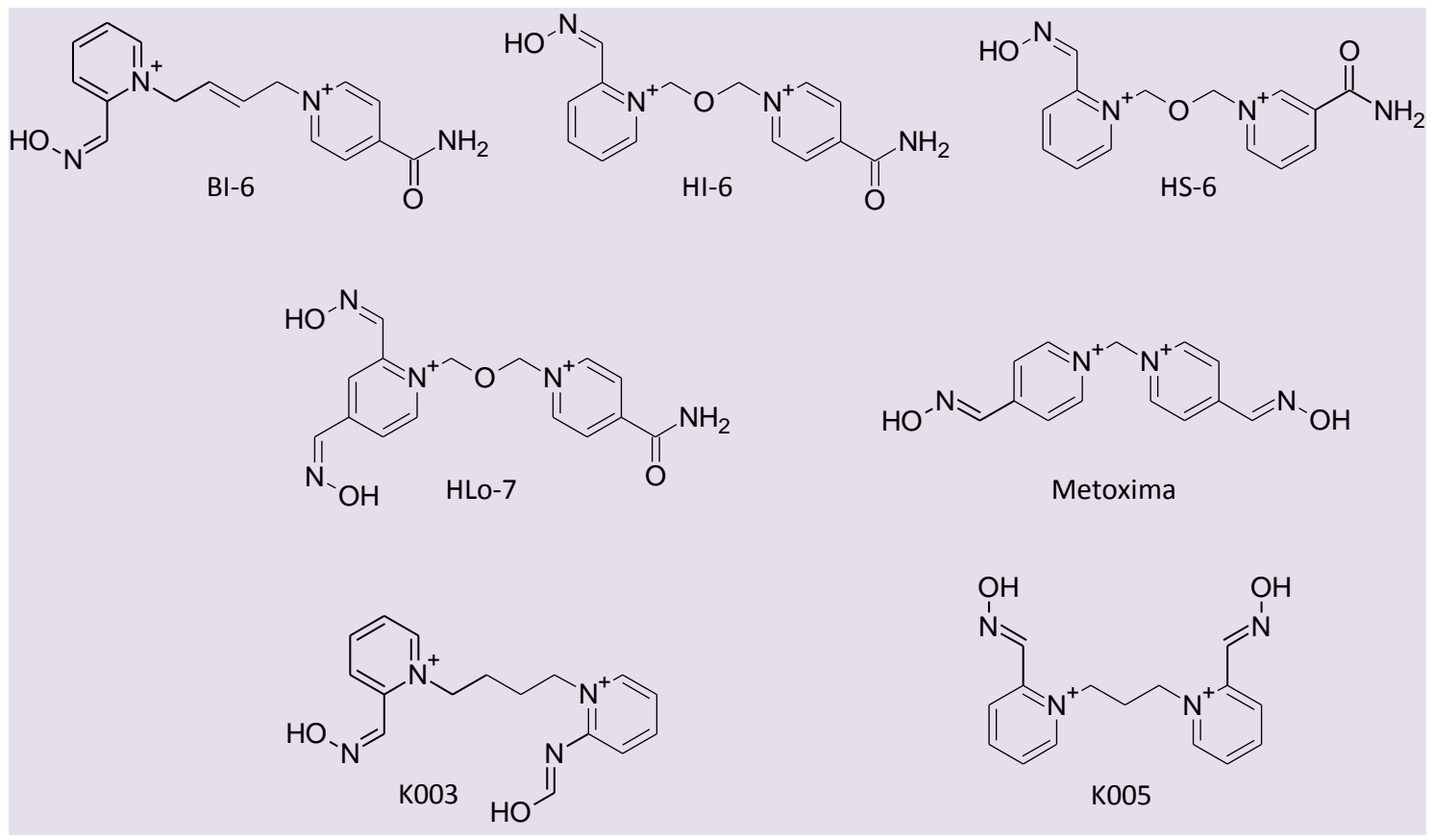

Figura 3. Oximas estudadas

\subsection{Os métodos híbridos $\mathrm{QM} / \mathrm{MM}$}

Devido ao grande número de átomos em proteínas e ao fato das reações envolverem a quebra e a formação de ligações químicas, a catálise enzimática é um grande desafio para a química computacional. ${ }^{50}$

Métodos quanto-mecânicos de alto nível teórico são limitados à aplicação em sistemas com número relativamente pequeno de átomos. A combinação dos métodos $\mathrm{QM} / \mathrm{MM}$ estende o domínio dos cálculos QM a macromoléculas. A estratégia básica nessa metodologia, introduzida por Warshel e Levitt (1976), ${ }^{51}$ pode ser descrita da seguinte forma: assume-se que o sistema molecular pode ser dividido em dois subsistemas: as regiões QM e MM.

A região $M M$ contém todos os átomos que são tratados explicitamente no cálculo. Na região $Q M$, os átomos são representados por núcleos e elétrons e a superfície de potencial é construída dentro da aproximação de Born-Oppenheimer. ${ }^{52}$
2.2.1. Estudos das interações entre a oxima BI-6 e a AChE inibida por diferentes OPs

Os cálculos de mecanismo de reação e DFT foram realizados nos programas Spartan08 ${ }^{{ }^{47}}$ e Gaussian09 ${ }^{\circledR}{ }^{53}$ respectivamente. As regiões QM foram cortadas a partir dos resultados de ancoramento molecular no software SPDBViewer. $^{49}$ Esta região consiste no mecanismo de reação dos resíduos pertencentes à tríade catalítica (Ser203, Glu334 e His447), resíduos que sofreram interações de hidrogênio com a oxima e o cofator. Todos os estados de transição (ET), intermediários e precursores envolvidos foram calculados e a caracterização destes ET foi realizada pelo cálculo da frequência imaginária. Cada confôrmero foi totalmente otimizado em ambos os níveis, PM3 e DFT com os algoritmos de gradientes conjugados e quasi-Newton-Raphson. A geometria final foi obtida pelo método DFT utilizando B3LYP/6-31G+(d,p). ${ }^{54}$ 
2.2.2. Estudos por $Q M / M M$ das interações entre diferentes oximas e a AChE inibida por ciclosarin

A metodologia utilizada para esses estudos é similar a do item anterior com pequenas diferenças. Nesse caso os cálculos QM foram desenvolvidos nos pacotes Spartan08 $8^{{ }^{47}}$ e Gaussian $98,{ }^{55}$ as regiões QM foram delimitadas dentro de uma esfera com raio entre 9 a 15 Á centrada em cada oxima e cada confôrmero foi totalmente otimizado em nível DFT com B3LYP/6-31G. ${ }^{54}$

\section{Resultados e Discussão}

$\mathrm{Na}$ tabela 1 são apresentados os resultados experimentais, os valores de energia de interação intermolecular e os resíduos que interagiram com a oxima $\mathrm{BI}-6$ para os complexos MmAChE-OP nos estudos por ancoramento.

Tabela 1. Resultados de ancoramento da oxima BI-6 na MmAChE.

\begin{tabular}{ccccc}
\hline Complexo & Resíduo & $\begin{array}{c}\Delta \mathrm{E}^{*} \\
\left(\mathrm{kcal} \mathrm{mol}^{-1}\right)\end{array}$ & $\begin{array}{c}\text { Energia H-bond } \\
\left(\mathbf{k c a l ~ m o l}^{-1}\right)\end{array}$ & $\begin{array}{c}\text { Valores experimentais do } \\
\text { processo de reativação (\%) }\end{array}$ \\
\hline $\begin{array}{c}\text { AChE- } \\
\text { ciclosarin }\end{array}$ & $\begin{array}{c}\text { Tyr124 } \\
\text { Tyr124 }\end{array}$ & $-159,44$ & $-5,52$ & 1 \\
Sistema 1 & Tyr124 & & & 9 \\
\hline AChE-soman & $\begin{array}{c}\text { Tyr124 } \\
\text { Tyr124 }\end{array}$ & $-152,14$ & $-9,37$ & 22 \\
Sistema 2 & $\begin{array}{c}\text { Tyr124 } \\
\text { Glu285 }\end{array}$ & & & \multirow{2}{*}{32} \\
\hline AChE-tabun & Tyr124 & $-155,54$ & $-4,34$ & \\
Sistema 3 & Tyr124 & & & \\
\hline AChE-sarin & Tyr124 & & & \\
Sistema 4 & Tyr124 & $-152,21$ & $-3,61$ & \\
\hline
\end{tabular}

${ }^{*} \Delta \mathrm{E}=$ Energia de interação intermolecular

É perceptível que não há uma diferença significativa nos valores de energia de interação intermolecular. O que já era esperado, uma vez que foi realizado estudo de re-docking com a mesma oxima, assim as interações entre a oxima e a enzima não sofreriam grandes mudanças. Com exceção do sistema 2 (que também interagiu com o resíduo Glu285) todos os outros apresentaram interações com a Tyr124 (Figura 4). Dessa forma, o número de interações com esse resíduo é capaz de determinar o modo de interação com a oxima BI-6. 

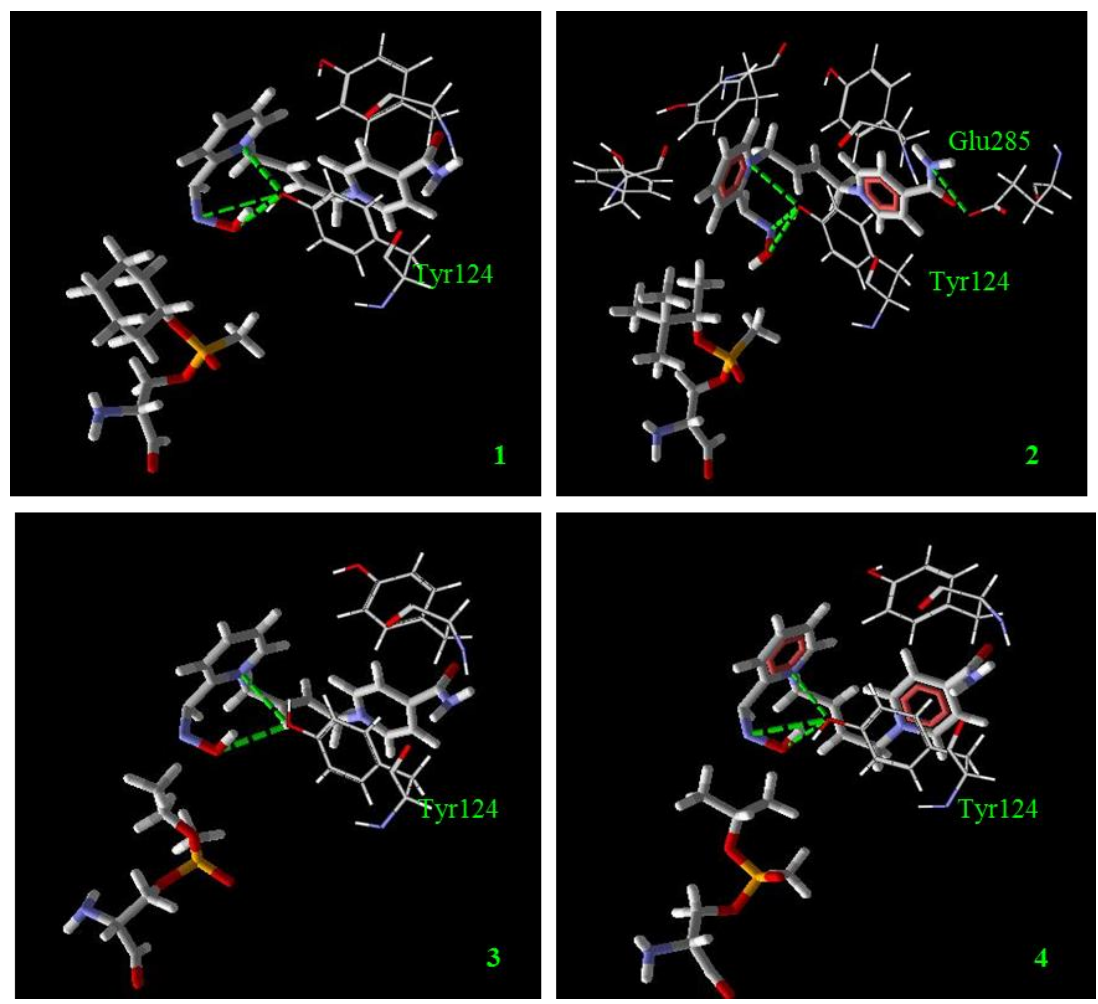

Figura 4. Interações observadas em cada sistema estudado. 1 - sistema AChE-ciclosarin, 2 sistema AChE-soman, 3 - sistema AChE-tabun e 4 - sistema AChE-sarin

Observou-se também uma possível interação do tipo empilhamento $\pi-\pi$ ("stacking") entre o anel piridina de $\mathrm{BI}-6$ e a Tyr124. Interações de longo alcance podem estar contribuindo com estabilidade extra para o estado de transição (ET). Essa interação foi observada em outro estudo realizado por nosso grupo de pesquisa. ${ }^{23}$

É importante notar que os resultados teóricos de energia de interação não explicam bem os resultados experimentais. Baseando-se nesse fato, foram realizados cálculos teóricos para determinar a $\Delta \Delta \mathrm{E}^{\#}$ relativa em cada sistema estudado nos quais foram comparados os valores de $\Delta \mathrm{E}^{\#}$ entre o ET e a configuração inicial de cada sistema. Assim obtivemos a tendência de reativação de cada oxima, evitando-se assim o cálculo direto dos valores absolutos de energia.

Numerosos estudos têm investigado o mecanismo de ação das oximas nas últimas décadas, e há evidências convincentes da ação das oximas, principalmente como reativadores de $\mathrm{AChE}^{3,5} \mathrm{O}$ processo de reativação acontece via duas etapas de reação (Equação 5). Primeiro há a formação de um complexo totalmente reversível AChEfosfil (no caso de inibição por OP) do tipo Michaelis (ET penta-coordenado), seguido do deslocamento do fosfil do ET. O $K_{\mathrm{R}}$ aproximase, então, da constante de dissociação a qual é inversamente proporcional à afinidade da oxima pela AChE fosforilada. A reatividade da oxima pode ser expressa em razão da constante de velocidade $k_{\mathrm{R}}$.

$$
\mathrm{EI}+\mathrm{Ox} \stackrel{K_{\mathrm{R}}}{\rightleftharpoons} \mathrm{EIOx} \stackrel{k_{\mathrm{R}}}{\longrightarrow} \mathrm{E}+\mathrm{I}-\mathrm{Ox}
$$

Eq. 5 
$\mathrm{Na}$ Tabela 2 estão reportados os parâmetros cinéticos $\Delta \Delta \mathrm{E}^{\#}$ preditos por cálculos teóricos, os valores experimentais e as respectivas frequências imaginárias, que caracterizam as estruturas de cada ET.

Tabela 2. Resultados experimentais, energia intermolecular e energia de ativação relativa de $\mathrm{BI}-6$ com 4 diferentes agentes de nervos

\begin{tabular}{ccccc}
\hline Sistema & $\begin{array}{c}\text { \% de } \\
\text { reativação }\end{array}$ & $\Delta \mathrm{E}\left(\mathrm{kcal} \mathrm{mol}^{-1}\right)$ & $\Delta \Delta \mathrm{E}^{\# \mathrm{a}}\left(\mathrm{kcal} \mathrm{mol}^{-1}\right)$ & Frequência $\left(\mathrm{cm}^{-1}\right)$ \\
\hline AChE-ciclosarin & 1 & $-159,44$ & 0,00 & $\mathrm{i} 97,88$ \\
AChE-soman & 9 & $-152,14$ & $-18,16$ & $i 95,83$ \\
AChE-tabun & 22 & $-155,54$ & $-21,95$ & $\mathrm{i} 100,25$ \\
AChE-sarin & 32 & $-152,21$ & $-28,22$ & $i 97,95$ \\
\hline
\end{tabular}

${ }^{\mathrm{a}} \Delta \Delta \mathrm{E}^{\#}$ (usando o complexo AChE-ciclosarin como referência) $=\Delta_{\mathrm{LIG} 2^{-}} \Delta_{\mathrm{LIG1}}$

Como observado anteriormente, as energias de interação dos cálculos de ancoramento são muito semelhantes entre os sistemas estudados. Portanto, os dados experimentais não podem ser racionalizados apenas com os valores de $\Delta \mathrm{E}$. Voltando-nos agora para o mecanismo de reação entre oxima e os diferentes OPs, os valores de $\Delta \Delta \mathrm{E}^{\#}$ podem racionalizar, porém em parte, os resultados experimentais $\left(R^{2}=0.75\right.$, Figura 5$)$. Espera-se que uma melhor correlação entre a teoria e o experimento possa ser alcançada quando outros efeitos relacionados com o processo de interação forem incluídos.

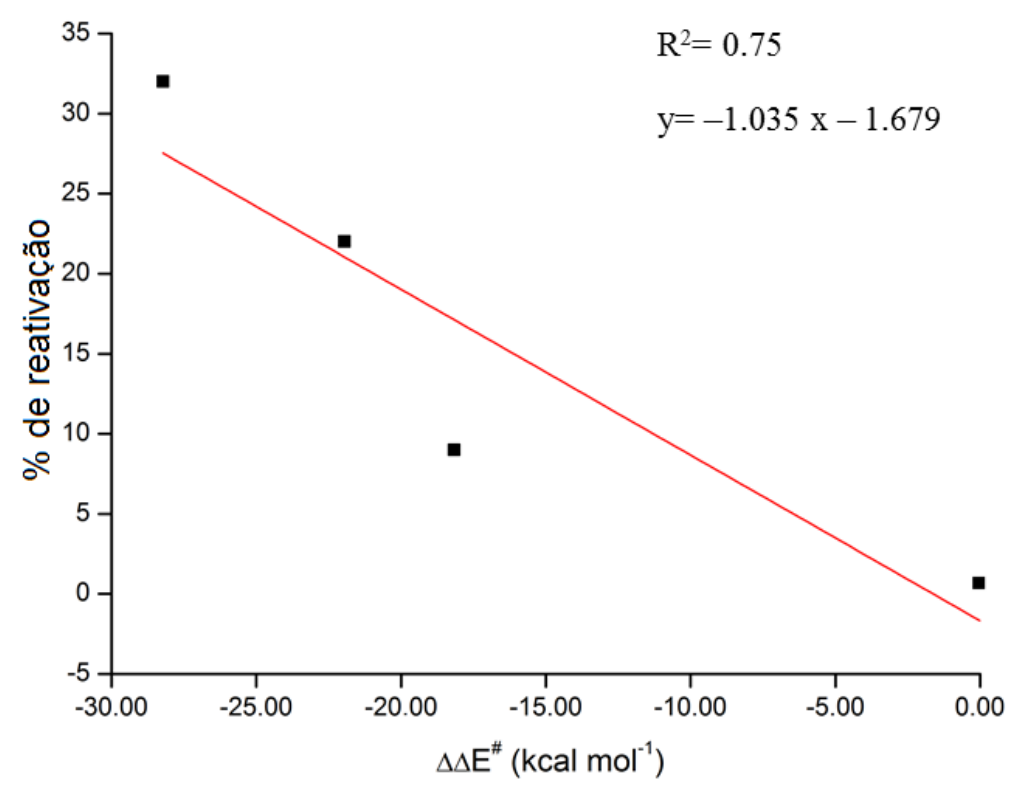

Figura 5. \% de reativação versus $\Delta \Delta \mathrm{E}^{\#}$ para os sistemas estudados

De fato, o processo de reativação depende de ambos os passos: 1) a interação da oxima com a enzima inibida e 2 ) a reação química entre a oxima e o neurotóxico. A partir dos dados apresentados na Tabela 2, um modelo matemático foi construído 
usando o método de regressão linear múltipla (MLR), resultando na Equação 4 para prever o \% de reativação dos sistemas estudados.
Os valores teóricos para o \% de reativação obtidos da Equação 4 mostraram uma boa correlação com os resultados experimentais $\left(R^{2}=0.90\right)$. A mesma correlação não foi observada para os parâmetros cinéticos e de interação, separadamente $\left(R^{2}=0.07\right.$ e $R^{2}=$ 0.75, respectivamente). Assim, nossos resultados teóricos colocam em evidência que a interação e o mecanismo de reação têm papel importante no processo de reativação.

Pela equação 4 percebe-se que valores de energia de interação mais baixos levam a um maior \% de reativação. Vale ressaltar que os valores para os coeficientes $\Delta \mathrm{E}$ e $\Delta \Delta \mathrm{E}^{\#}$ na equação 4 são semelhantes, mas os parâmetros cinéticos são mais sensíveis a pequenas variações.
3.2. Estudos das interações entre diferentes oximas e a AChE inibida por ciclosarin

Para avaliar o poder de reativação das oximas, é necessário comparar as energias de interação entre os resíduos presentes no sítio ativo da MmAChE e o ciclosarin. Isso possibilitará o melhor entendimento dos modos de interação e fatores responsáveis pelas atividades de cada oxima. Na Tabela 3 e na Figura 7 são apresentados os valores experimentais de $\mathrm{pK}_{\mathrm{R}}$ e energia de interação $(\Delta E)$ para as oximas em estudo.

Tabela 3. Valores experimentais de $\mathrm{pk}_{\mathrm{R}}$ e energia de interação (EI) para as oximas estudadas

\begin{tabular}{|c|c|c|c|}
\hline Oxima & $\mathrm{pK}_{\mathrm{R}}^{*}\left(\mathrm{mmol} \mathrm{L}^{-1}\right)$ & $\Delta \mathrm{E}^{* *}\left(\mathrm{kcal}^{\mathrm{m}} \mathrm{mol}^{-1}\right)$ & Resíduos \\
\hline K005 & 5.30 & -143.48 & $\begin{array}{c}\text { Phe338/ } \\
\text { Phe295/Tyr124/Arg296/Trp286 }\end{array}$ \\
\hline HI-6 & 4.92 & -142.98 & $\begin{array}{c}\text { Tyr124/Tyr124/Tyr124/Tyr124/ } \\
\text { Ser298/Ser298 }\end{array}$ \\
\hline Metoxima & 4.82 & -142.32 & $\begin{array}{l}\text { Thr83/Tyr337/Tyr337/Tyr341/ } \\
\text { Tyr124/Tyr124/Ser298/Ser298 }\end{array}$ \\
\hline K033 & 4.70 & -142.86 & $\begin{array}{c}\text { Trp286/Arg296/Arg296/Phe295/ } \\
\text { Tyr124/Tyr124 }\end{array}$ \\
\hline HS-6 & 4.35 & -142.67 & Tyr124/Tyr124/Phe295 \\
\hline HLÖ-7 & 3.00 & -141.91 & $\begin{array}{c}\text { Arg296/Tyr124/Tyr124/Tyr124 } \\
\text { Tyr124 }\end{array}$ \\
\hline $\mathrm{BI}-6$ & 1.00 & -140.21 & Glu285/Tyr124 \\
\hline
\end{tabular}

\footnotetext{
*Dados experimentais; ${ }^{* *}$ Dados teóricos.
} 


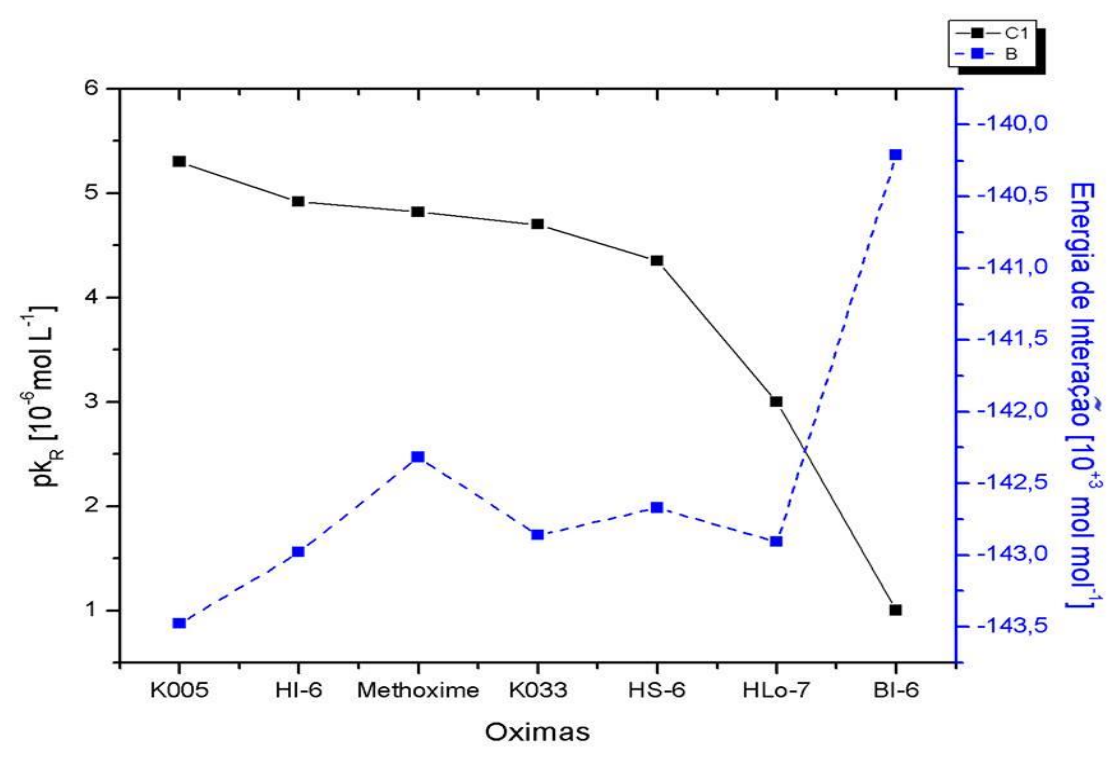

Figura 7. Valores da constante experimental de dissociação do complexo reativador-enzima inibida $\left(\mathrm{pK}_{\mathrm{R}}=-\log \mathrm{K}_{\mathrm{R}}, \mu \mathrm{mol} \mathrm{L} \mathrm{L}^{-1}\right)^{48}$ e energia de interação intermolecular $\left(\mathrm{kcal} \mathrm{mol}{ }^{-1}\right)$ proteína/inibidores obtida do ancoramento

Podemos concluir pela Figura 7, que o maior valor de $\mathrm{pK}_{\mathrm{R}}$ corresponde a um menor valor de energia de interação intermolecular. Nesse sentido, a oxima K005 apresenta uma maior afinidade pelo sítio ativo da MmAChE. Em contrapartida, a oxima BI-6 apresenta menor afinidade pelo sítio da mesma enzima.

Foi observado também que a $\mathrm{BI}-6$ faz o menor número de ligações de hidrogênio enquanto que HS-6 apresenta 3 interações, KO05 e HLÖ-7 apresentam 5, K033 e HI-6 apresentam 6 e a metoxima apresenta 8 interações com os aminoácidos do sítio ativo.

É importante ressaltar que uma mínima mudança na estrutura molecular dos reativadores é capaz de alterar significativamente sua reatividade e, consequentemente, sua capacidade de reativar a enzima.

Após a realização do estudo por QM/MM obtivemos os valores de $\Delta \Delta \mathrm{E}^{\#}$ e frequência imaginária, para as oximas estudadas, descritos na Tabela 4.

A Tabela 4 mostra que as reativações, pelas oximas Hlö-7 e HI-6, da MmAChE inibida por ciclosarin apresentaram a menor e a maior barreira de energia, respectivamente. Uma explicação plausível é que a estabilização do ET seja favorecida por ligações hidrogênio com os resíduos próximos ao sítio ativo. Esses aminoácidos (Tyr124, Trp286, Phe295, Arg296 e Phe338) orientam a oxima em direção à geometria adequada para o ET. Nessa conformação, as oximas interagem mais fortemente com 0 ciclosarin, aumentando assim a probabilidade de formação dos produtos.

As interações de longo alcance entre as oximas e outros aminoácidos também podem ocorrer, como interações cátion- $\pi$ com Tyr34, Phe295, Tyr337 e Phe338, interações eletrostáticas com Glu285 e, supreendentemente, interações de empilhamento $\pi$ entre $o$ anel piridina da oxima e os resíduos Tyr124 e Trp286, que ocorrem na geometria do ET.

A oxima K005 é considerada um dos melhores reativadores da AChE inibida por ciclosarin e apresenta bons resultados de $K_{R}$ (Figura 7) e, portanto, uma alta afinidade pela enzima intacta (Tabela 3). Porém, vale ressaltar que o excesso dessa oxima também pode inibir a enzima reativada. ${ }^{48,56}$ 
Tabela 4. Energias de ativação relativa dos ETs e parâmetros cinéticos das oximas estudadas

\begin{tabular}{cccc}
\hline Oxima & $\mathrm{kr}^{\mathrm{a}} / \mathrm{min}^{-1}$ & $\Delta \Delta \mathrm{E}^{\mathrm{\# b}} /\left(\mathrm{kcal} \mathrm{mol}^{-1}\right)$ & Frequência $/ \mathrm{cm}^{-1}$ \\
\hline HI-6 & 0,350 & 220,85 & $\mathrm{i} 5,15$ \\
\hline BI-6 & 0,150 & 170,43 & $\mathrm{i} 64,21$ \\
\hline metoxima & 0,240 & 132,00 & $\mathrm{i} 16,59$ \\
\hline HS-6 & 0,156 & 131,12 & $\mathrm{i} 13,68$ \\
\hline K033 & 0,095 & 82,34 & $\mathrm{i} 5,21$ \\
\hline K005 & 0,010 & 31,96 & $\mathrm{i} 74,20$ \\
\hline HLö-7 & 0,008 & 0,00 & $\mathrm{i} 2,31$ \\
\hline
\end{tabular}

.$^{\mathrm{a}}$ Constante de velocidade de reativação; $\Delta \Delta \mathrm{E} "=\mathrm{E}_{\mathrm{uc}}-\mathrm{E}_{\mathrm{H} \iota \mathrm{r}}$.

\section{Considerações finais}

$\mathrm{Na}$ pesquisa 1, observou-se que $\mathrm{o}$ processo de interação de $\mathrm{Bl}-6$ é favorecido por interações com Tyr124 e Glu285. Ao analisar os resultados de energia de interação obtidos, percebe-se que eles não explicam os valores experimentais. Assim, os cálculos teóricos de energia de ativação foram feitos e mostraram boa correlação com os valores de percentagem de reativação. Porém, ao correlacionar as energias de interação e de ativação relativa, obtivemos um $R^{2}=0,90$ o que nos leva a concluir que nossas descobertas teóricas evidenciam que tanto a etapa de interação quanto a de reação química exercem um papel importante no processo de reativação.

$\mathrm{Na}$ pesquisa 2, observou-se que o processo de interação das oximas é favorável com os resíduos Tyr124, Trp286, Phe295 e Arg296. Já com relação à reação química, notou-se que os aminoácidos Tyr124, Trp286 e Arg296 são responsáveis pela estabilização do ET. Assim, acredita-se que os parâmetros cinéticos calculados podem ser úteis para o projeto e seleção de uma nova oxima mais eficiente. É importante destacar que observamos, em ambas as pesquisas, que o número de ligações hidrogênio com o resíduo Tyr124 é uma característica fundamental para determinar o modo de interação das oximas.

Os estudos por modelagem molecular das oximas como reativadores da AChE, disponíveis na literatura, apontam muitos pontos importantes a serem considerados para o melhor entendimento do mecanismo de ação e posterior projeção de um novo reativador de AChE. Nossos resultados mostraram uma boa correlação entre os dados teóricos e dados experimentais, fazendo com que a metodologia usada corrobore para a predição dos parâmetros cinéticos e termodinâmicos para reativadores da AChE.

\section{Agradecimentos}

Os autores agradecem ao Conselho Nacional de Desenvolvimento Científico e Tecnológico (CNPq), a Fundação de Amparo à Pesquisa do Estado do Rio de Janeiro (FAPERJ), a Fundação de Amparo à Pesquisa de Minas Gerais (FAPEMIG), a Coordenação de Aperfeiçoamento de Pessoal de Nível Superior / Ministério da Defesa (CAPES / MD) (Edital PRODEFESA 2008) pelo apoio financeiro, e ao Instituto Militar de Engenharia (IME) e a Universidade Federal de Lavras (UFLA) por fornecerem a infraestrutura física e o espaço de trabalho. 


\section{Referências Bibliográficas}

${ }^{1}$ Thant, U. As armas químicas e bacteriológicas (biológicas) e os efeitos de seu possível emprego. Departamento de Assuntos Políticos e do Conselho de Segurança. Relatório do Secretário-Geral das Nações Unidas № E. 69.I.24.

${ }^{2}$ Amitai, G.; Murata, H.; Andersen, J. D.; Koepsel, R. R.; Russell, A. J. Decontamination of chemical and biological warfare agents with a single multi-functional material. Biomaterials 2010, 31, 4417. [CrossRef]

${ }^{3}$ Taylor, C. L.; Taylor Júnior, L. B. Chemical and biological warfare. Franklin Watts: New York, 1992.

${ }^{4}$ Herrmann, H. W.; Henins, I.; Park, J.; Selwyn, G. S. Decontamination of chemical and biological warfare (CBW) agents using an atmospheric pressure plasma jet (APPJ). Physics Plasmas 1999, 6, 2284. [CrossRef]

${ }^{5}$ França, T. C. C.; Silva, G. R.; Castro, A. T. de. Defesa química: uma nova disciplina no ensino de química. Revista Virtual de Química 2010, 2, 84. [Link]

${ }^{6}$ França, T. C. C.; Castro, A. T.; Rennó, M. N.; Figueroa-Villar, J. D. A questão da defesa contra agentes de guerra biológica nas Forças Armadas e no Brasil. C\&T: Revista Militar de Ciência e Tecnologia 2008, 27, 56. [Link]

${ }^{7}$ Smart, J. K. Em Textbook of military medicine: medical aspects of chemical and biological warfare; Sidell, F. R.; Takafuji, E. T.; Franz, D. R., eds.; Office of The Surgeon General at TMM Publications Borden Institute: Washington, 1997, cap. 2.

${ }^{8}$ Delfino, R. T.; Ribeiro, T. S.; Figueroa-Villar, J. D. Organophosphorus compounds as chemical warfare agents: a review. Journal of the Brazilian Chemical Society 2009, 20, 387. [CrossRef]

${ }^{9}$ http://www.opcw.org/ acessado em 01 de março de 2014.

${ }^{10}$ Silva, G. R.; Borges Jr, I.; Figueroa-Villar, J. D.; Castro, A. T. Defesa química: histórico, classificação dos agentes de guerra e ação dos neurotóxicos. Química Nova 2012, 35, 2083. [CrossRef]
${ }^{11}$ Castro, A. T. Terrorismo químico e biológico: a ameaça do século XXI. C\&T: Revista Militar de Ciência e Tecnologia 2001, 18, 65. [Link]

${ }^{12}$ Colasso, C.; Azevedo, F. A. Riscos da utilização de Armas Químicas. Parte II Aspectos Toxicológicos. Revista Intertox de Toxicologia, Risco Ambiental e Sociedade 2012, 5, 7. [Link]

${ }^{13}$ Farias, R. Armas químicas. A ciência em mãos do mal, Plaza y Valdés Eds: Madrid, 2004.

${ }^{14}$ Patocka, J.; Cabal, J.; Kuca, K.; Jun, D. Oxime reactivation of acetylcholinesterase inhibited by toxic phosphorus esters: in vitro kinetics and thermodynamics. Journal of Applied Biomedicine 2005, 3, 91. [Link]

${ }^{15}$ Eddleston, M.; Szinicz, L.; Eyer, P.; Buckley, $N$. Oximes in acute organophosphorus pesticide poisoning: a systematic review of clinical trials. The Quarterly Journal of Medicine 2002, 95, 275. [CrossRef] [PubMed] ${ }^{16}$ Dougin, E. Syrian gas attack reinforces need for better anti-sarin drugs. Nature Medicine 2013, 19, 1194. [CrossRef]

${ }^{17}$ Kuca, K.; Cabal, J.; Jun, D.; Hrabinova, M. Potency of five structurally different acetylcholinesterase reactivators to reactivate human brain cholinesterases inhibited by cyclosarin Clinical Toxicology 2007, 45, 512. [CrossRef]

${ }^{18}$ Saint-André, G.; Kliachyna, M.; Kodepelly, S.; Louise-Leriche, L.;Gillon, E.; Renard, P.-Y.; Nachon, F.; Baati, R.; Wagner, A. Design, synthesis and evaluation of new $\alpha$ nucleophiles for the hydrolysis of organophosphorus nerve agents: application to the reactivation of phosphorylated acetylcholinesterase. Tetrahedron 2011, 67, 6352. [CrossRef]

${ }^{19}$ Matatagui, D.; MArtí, J.; Fernández, J. L.; Fontecha, J. I.; Gutiérrez, J.; Gràcia, I.; Cané, C.; Horrilo, M. C. Chemical warfare agents simulants detection with an optimized SAW sensor array. Sensors and Actuators B: Chemical 2011, 154,199. [CrossRef]

${ }^{20}$ Matos, K. S.; Dissertação de Mestrado, Universidade Federal de Lavras, Lavras, 2012. [Link] 
${ }^{21}$ Soukup, O.; Kristofikova, Z, Proska, J.; Tobin, G.; Patocka, J.; Marek, J.; Jun, D.; Fusek, J.; Ripova, D.; Kuca, K. Novel acetylcholinesterase reactivator $\mathrm{K} 112$ and its cholinergic properties. Biomedicine \& Pharmacotherapy 2010, 64, 541. [CrossRef]

22 Wang, J.; Gu, J.; Leszczynski, J.; Feliks, M.; Sokalski, W. A. Oxime-induced reactivation of sarin-inhibited ache: a theoretical mechanisms study. The Journal of Physical Chemistry B 2007, 111, 2404. [CrossRef]

23 Matos, K. S.; Mancini, D. T.; da Cunha, E. f. F.; Kuca, K.; França, T. C. C.; Ramalho, T. C. Molecular aspects of the reactivation process of acetylcholinesterase inhibited by cyclosarin. Journal of the Brazilian Chemical Society 2011, 22, 1999. [CrossRef]

${ }^{24}$ Worek, F.; Bierwisch, A.; Wille, T.; Koller, M.; Thiermann, $H$. Kinetic interactions of a homologous series of bispyridinium monooximes (HGG oximes) with native and phosphonylated human acetylcholinesterase. Toxicology Letters 2012, 212, 29. [CrossRef]

${ }^{25}$ Albuquerque E. X.; Pereira E. F.; Aracava Y.; Fawcett W. P.; Hamilton T. A.; Kan R. K.; Romano J. A.; Adler M. Effective countermeasure against poisoning by organophosphorus insecticides and nerve agents. Proceedings of the National Academy of Sciences of the USA 2006, 103, 13220. [CrossRef]

${ }^{26}$ Hörnberg, A.; Tunemalm, A.; Ekström, F. Crystal structures of acetylcholinesterase in complex with organophosphorus compounds suggest that the acyl pocket modulates the aging reaction by precluding the formation of the trigonal bipyramidal transition state. Biochemistry 2007, 46, 4815. [CrossRef]

27 Soreq, H.; Ben-Aziz, R.; Prody, C. A.; Seidman, S.; Gnatt, A.; Neville, L.; LiemanHurwitz, J.; Lev-Lehman, E.; Ginzberg, D.; Lipidot-Lifson, Y. Molecular cloning and construction of the coding region for human acetylcholinesterase reveals a $\mathrm{G}+\mathrm{C}$-rich attenuating structure. Proceedings of the National Academy of Sciences of the USA 1990, 87, 9688. [CrossRef]

${ }^{28}$ Gonçalves, A. S.; Tese de Doutorado, Universidade Federal do Rio de Janeiro, Rio de Janeiro, 2009. [Link]
${ }^{29}$ Quinn, D. M. Acetylcholinesterase: enzyme structure, reaction dynamics, and virtual transition states. Chemical Reviews 1987, 87, 955. [CrossRef]

${ }^{30}$ Bourne C. R.; Bunce R. A.; Bourne P. C.; Berlin K. D.; Barrow E. W.; Barrow W. W. Crystal Structure of Bacillus anthracis Dihydrofolate Reductase with the Dihydrophthalazine-Based Trimethoprim Derivative RAB1 Provides a Structural Explanation of Potency and Selectivity. Antimicrobial Agents and Chemotherapy 2009, 53, 3065. [CrossRef]

${ }^{31}$ Worek, F.; Koller, M.; Thiermann H.; Szinicz, I. Diagnostic aspects of organophosphate poisoning. Toxicology 2005, 214, 182. [CrossRef]

32 Dickoff, D. J.; Gerber, O.; Turovsky, Z. Delayed neurotoxicity after ingestion of carbamate pesticide. Neurology 1987, 37, 1229. [CrossRef]

${ }^{33}$ Rosman, Y.; Makarovsky, I.; Bentur, Y.; Shrot, S.; Dushnistky, T.; Krivoy, A. Carbamate poisoning: treatment recommendations in the setting of a mass casualties event. The American Journal of Emergency Medicine 2009, 27, 1117. [CrossRef]

${ }^{34}$ Stojiljkovic, M. P.; Jokanovic, M. Pyridinium oximes: rationale for their selection as causal antidotes against organophosphate poisonings and current solutions for autoinjectors. Arhiv za Higijenu Rada I Toksikologiju 2006, 57, 435. [PubMed]

${ }^{35}$ Kassa, J.; Karasova, J. Z.; Sepsova, V.; Caisberger, $F$. The benefit of combinations of oximes for the reactivating and therapeutic efficacy of antidotal treatment of sarin poisoning in rats and mice. Basic \& Clinical Pharmacology \& Toxicology 2012, 22, 260. [CrossRef]

${ }^{36}$ Laufer, R.; Kalasz, H.; Musilek, K.; Szegi, P.; Darvas, F.; Kuca, K.; Tekes, K. Synthesis, antidotal effects and HPLC behavior of some novel pyridinium aldoximes. Current Organic Chemistry 2010, 14, 447. [CrossRef]

${ }^{37}$ Giacoppo, J. O. S.; França, T. C. C.; Kua, K. da Cunha, E. F. F.; Abagyan, R.; Mancini, D.T.; Ramalho, T. C. Submetido ao Journal of the Brazilian Chemical Society, 2013. 
${ }^{38}$ Silveira, R. L. V. de A. Fitotoxicidade de glifosato em eucalyptus. Addubare 2003, 9, 4. [Link]

39 D'Alfonso, G.; Tramontano, A; Lahn, A. Structural conservation in single-domain proteins: implications for homology modeling. Journal of Structural Biology 2001, 134, 246. [CrossRef]

${ }^{40}$ Souza, T. C. S.; Josa, D.; Ramalho, T. C.; Caetano, M. S.; da Cunha, E. F. F. Molecular modelling of Mycobacterium tuberculosis acetolactate synthase catalytic subunit. Molecular Simulation 2008, 34, 707. [CrossRef]

${ }^{41}$ Thomsen, R.; Christensen, M. H. MolDock: a new technique for high accuracy molecular docking. Journal of Medicinal Chemistry 2006, 49, 3315. [CrossRef]

${ }^{42}$ Ramalho, T. C.; Caetano, M. S.; da Cunha, E. F.; Souza, T. C.; Rocha, M. V. Construction and assessment of reaction models of Class I EPSP synthase: molecular docking and density functional theoretical calculations. Journal of Biomolecular Structure and Dynamics 2009, 27, 95. [CrossRef]

${ }^{43}$ Ogungbe, I. V.; Setzer, W. N. Comparative molecular docking of antitrypanosomal natural products into multiple trypanosoma brucei drug targets. Molecules 2009, 14, 1513. [CrossRef]

${ }^{44}$ Mizutani, M. Y.; Tomioka, N.; Itai, A. Rational automatic search method for stable docking models of protein and ligand. Journal of Molecular Biology 1994, 243, 310. [CrossRef]

${ }^{45}$ Kuca, K.; Cabal, J. Evaluation of newly synthesized reactivators of the brain cholinesterase inhibited by sarin nerve agent. Toxicology Mechanisms and Methods 2005, 15, 247. [CrossRef]

${ }^{46}$ Berman, H. M.; Westbrook, J.; Feng, Z.; Gilliland, G.; Bhat, T. N.; Weissig, H.; Shindyalov, I. N.; Bourne, P. E. The Protein
Data Bank. Nucleic Acids Research 2000, 28, 2352. [CrossRef]

${ }^{47}$ Hehre, W. J.; Deppmeier, B. J.; Klunzinger, P. E.; PC Spartan Pro, 1999 Wavefunction: Irvine, $C A$.

${ }^{48}$ Kassa, J.; Kuca, K.; Bartosova, L.; Kunesova, G. The development of new structural analogues of oximes for the antidotal treatment of poisoning by nerve agents and the comparison of their reactivanting and therapeutic efficacy with currently available oximes. Current Organic Chemistry 2007, 11, 267. [CrossRef]

${ }^{49}$ Guex, N.; Peitsch, M. C. SWISS-MODEL and the Swiss-PdbViewer: an environment for comparative protein modeling. Electrophoresis 1997, 18, 2714. [CrossRef]

${ }^{50}$ Borman S. Much ado about anzyme mechanisms. Chemical and Engineering News 2004, 82, 35. [Link]

${ }^{51}$ Warshel, A.; Levitt, M. Theorical studies of enzymatic reactions-dielectric, electrostatic and steric stablilization of carbonium-ion in reaction of lysozyme. Journal Molecular Biology 1976, 103, 227. [CrossRef]

${ }^{52}$ Szabo, A.; Ostlund, N. S.; Modern quantum chemistry: Introdution to advanced electronic structure theory, Courier Dover: New York, 1996. [Link]

${ }^{53}$ Frisch M, J.; et al. Gaussian 09, Gaussian, Inc., Wallingford, CT, 2009.

${ }^{54}$ Lee, C.; Yang, W.; Parr, R. G. Development of the Colle-Salvetti correlation-energy formula into a functional of the electron density. Physical Review B 1988, 37, 785. [Link]

${ }^{55}$ Frisch, M. J.; et al. Gaussian 03, Gaussian, Inc., Wallingford, CT, 2004.

${ }^{56}$ Ramalho, T. C; França, T. C. C.; Rennó, M. N.; Guimarães, A. P.; Cunha, E. F. F.; Kuča, K. Development of new acetylcholinesterase reactivators: Molecular modeling versus in vitro data. Chemico-Biological Interactions 2010, 185, 73. [CrossRef] 